\title{
A Multi-Arm Phase I Study of the PI3K/mTOR Inhibitors PF-04691502 and Gedatolisib (PF-05212384) plus Irinotecan or the MEK Inhibitor PD-0325901 in Advanced Cancer
}

\author{
Zev A. Wainberg ${ }^{1} \cdot$ Maria Alsina $^{2} \cdot$ Heloisa P. Soares ${ }^{3}$ - Irene Braña ${ }^{4}$. \\ Carolyn D. Britten ${ }^{5}$. Gianluca Del Conte ${ }^{6} \cdot$ Patrick Ezeh $^{7} \cdot$ Brett Houk $^{8}$. \\ Kenneth A. Kern ${ }^{8} \cdot$ Stephen Leong $^{9} \cdot$ Nuzhat Pathan $^{8} \cdot$ Kristen J. Pierce $^{10}$. \\ Lillian L. Siu ${ }^{4}$. Jennifer Vermette $^{7}$. Josep Tabernero ${ }^{2}$
}

\begin{abstract}
Background This phase I, four-arm, open-label study (NCT01347866) evaluated the PI3K/mTOR inhibitors PF-04691502 (arms A, B) and gedatolisib (PF-05212384; arms $\mathrm{C}, \mathrm{D})$ in combination with the MEK inhibitor PD0325901 (arm A, D) or irinotecan $(\operatorname{arm~B}, \mathrm{C}$ ) in patients with advanced solid tumors.

Objectives Primary endpoint was dose-limiting toxicity with each combination. Secondary endpoints included safety, pharmacokinetics and preliminary antitumor activity.

Patients and Methods Dose escalation followed a $3+3$ design in arm $\mathrm{C}$ and a zone-based design in arm $\mathrm{D}$.

Results The PF-04691502 combination arms were closed prematurely due to low tolerability, and the maximum tolerated doses (MTDs) were not determined for either arm. The MTD for the combination of gedatolisib with irinotecan $180 \mathrm{mg} / \mathrm{m}^{2}$ was estimated to be $110 \mathrm{mg}$ weekly
\end{abstract}

Electronic supplementary material The online version of this article (https://doi.org/10.1007/s11523-017-0530-5) contains supplementary material, which is available to authorized users.

Zev A. Wainberg

ZWainberg@mednet.ucla.edu

1 David Geffen School of Medicine at UCLA, 2020 Santa Monica Blvd, Suite 600, Los Angeles, CA 90404, USA

2 Vall d'Hebron University Hospital and Institute of Oncology (VHIO), Universitat Autònoma de Barcelona, Vall d'Hebron 119-129, 08035 Barcelona, Spain

3 Moffitt Cancer Center, 12902 Magnolia Drive, Tampa, FL 33612, USA

4 Princess Margaret Cancer Centre, 610 University Avenue, Toronto M5G 2M9, Canada and for the combination with PD-0325901 was not reached at the highest dose evaluated (gedatolisib $154 \mathrm{mg}$ weekly). Plasma concentrations of gedatolisib were generally similar across dose groups in arm C (with irinotecan) and arm D (with PD-0325901). Frequent dose delays or dose reductions were required for both combinations, potentially preventing sustained therapeutic drug concentrations. Gedatolisib plus irinotecan produced a response rate of $\sim 5 \%$ and clinical benefit in $16 \%$ of patients with advanced colorectal cancer (progression-free survival, 2.8 months). Preliminary evidence of clinical activity was observed with gedatolisib plus PD-0325901 in patients with ovarian cancer (three partial responses, $n=5$ ) or endometrial cancer (one partial response, $n=1$ ) and KRAS mutations.

Conclusions Further evaluations of gedatolisib are warranted in patients with advanced solid malignancies.

5 Medical University of South Carolina, 39 Sabin St., MSC 635, Charleston, SC 29425, USA

6 IRCCS Ospedale S. Raffaele, via Olgettina 60, 20132 Milan, Italy

7 Pfizer Oncology, 300 Technology Square, Cambridge, MA 02139, USA

8 Pfizer Oncology, 10646 Science Center Drive, San Diego, CA 92121, USA

9 University of Colorado Denver School of Medicine, 13001 E 17th Place, Aurora, CO 80045, USA

10 Pfizer Oncology, 558 Eastern Point Road, Groton, CT 06340, USA 


\section{Key Points}

This study evaluated safety, pharmacokinetics, and preliminary antitumor activity of the PI3K/mTOR inhibitor gedatolisib plus irinotecan or the MEK inhibitor PD-0325901.

Gedatolisib combined with irinotecan had limited activity in patients with advanced colorectal cancer.

Antitumor activity was observed with gedatolisib in combination with PD-0325901 in patients with ovarian or endometrial cancer and KRAS mutations.

\section{Introduction}

The phosphatidylinositol 3-kinase (PI3K)/AKT/mammalian target of rapamycin (mTOR) and the mitogenactivated protein kinase (MAPK; RAS-RAF-MEKERK) pathways are aberrantly activated in many cancer cells $[1,2]$. RAS mutations are found in up to $30-40 \%$ of patients, whereas BRAF mutations occur in $\sim 10-12 \%$ and PI3K mutations in $10-18 \%$ of patients. Interestingly, RAS and PI3K mutations frequently coexist in the same tumor, but RAS and BRAF mutations are mutually exclusive [3]. It is known that these pathways converge at multiple points, and preclinical data suggest that blockade of these pathways may lead to upregulation of the other pathway via crosstalk [4-7]. Therefore, dual pathway blockade may be advantageous in inhibiting tumor growth, thus providing a rationale for evaluating the safety, tolerability and efficacy of combinations of PI3K and MEK inhibitors in patients with tumors bearing genetic aberrations of these pathways [8-12].

PF-04691502 is a potent dual inhibitor of both PI3K and mTOR (target of rapamycin complex [TORC] 1 and 2) kinase activity intended for once-daily oral dosing [13-15]. The maximum tolerated dose (MTD) for singleagent PF-04691502 in patients with solid malignancies was determined to be $8 \mathrm{mg}$ once daily [14]. Gedatolisib (PF-05212384) is a potent pan-class I isoform inhibitor of PI3K and mTOR (TORC1 and TORC2) kinase activity intended for once-weekly intravenous (IV) infusion [15-19]. The MTD for single-agent gedatolisib was estimated to be $154 \mathrm{mg}$ once weekly in patients with advanced solid tumors [19]. PD-0325901 is a potent, selective, nonATP-competitive, oral, small-molecule inhibitor of both MEK isoforms, MEK1 and MEK2 [20, 21]. This agent demonstrated antiproliferative and antitumor activity in preclinical models of cancer. The MTD for single-agent PD-0325901 was estimated to be $15 \mathrm{mg}$ orally twice a day (BID) [21].

Both gedatolisib and PF-04691502 inhibit PI3K and mTOR signaling in cell-based assays, resulting in antiproliferative activity in culture and antitumor activity in xenograft models $[13,16]$. Treatment with gedatolisib produced tumor growth inhibition or regression in multiple tumor types, including colon (HCT116), breast (MDA-MB-361, BT474) and lung cancer (H1975) xenograft models [16]. Preclinical data also support synergistic effects of PI3K inhibitors with irinotecan (topoisomerase I inhibitor) or PD-0325901 in solid tumors, as demonstrated with gedatolisib in experimental models of colorectal cancer (CRC) [16].

Aims of this multi-arm, dose-escalation, phase I study were to determine the MTD and/or recommended phase II dose for combination of the PI3K/mTOR inhibitors PF-04691502 and gedatolisib with irinotecan or the MEK inhibitor PD-0325901 and to assess their safety, pharmacokinetics (PK) and preliminary antitumor activity in patients with advanced solid tumors. By evaluating the two PI3K/mTOR inhibitors in parallel, with irinotecan or the MEK inhibitor, we sought to determine the most well-tolerated combination with which to move forward in the expansion phase.

\section{Patients and Methods}

\subsection{Study Design}

Study B1271002 was initially intended as a phase I, four-arm, open-label, multicenter, dose-escalation study of PF04691502 in combination with PD-0325901 (arm A) or irinotecan (arm B) and of gedatolisib in combination with irinotecan (arm C) or PD-0325901 (arm D) in patients with advanced solid tumors (Table 1). However, as a result of the emerging safety and antitumor activity data, the second study protocol amendment terminated enrollment to both PF04691502 arms. Arm A (PF-04691502 in combination with PD-0325901) was closed due to the frequent dose interruptions and dose reductions associated with treatment-related adverse events (AEs). Arm B (PF-04691502 in combination with irinotecan) was closed because of the more encouraging antitumor activity and safety profile observed in arm C (gedatolisib in combination with irinotecan). As a result of the better tolerability profile and higher antitumor activity seen in arm $\mathrm{C}$ compared with arm $\mathrm{B}$, a further arm, $\mathrm{D}$, was included to evaluate the combination of gedatolisib with PD0325901. Following dose escalation in stage 1, an expansion cohort (stage 2) was enrolled for arm C.

The primary objective of the study was to assess the safety and tolerability and define the MTD for each combination (primary endpoint: dose-limiting toxicity [DLT]). Secondary 


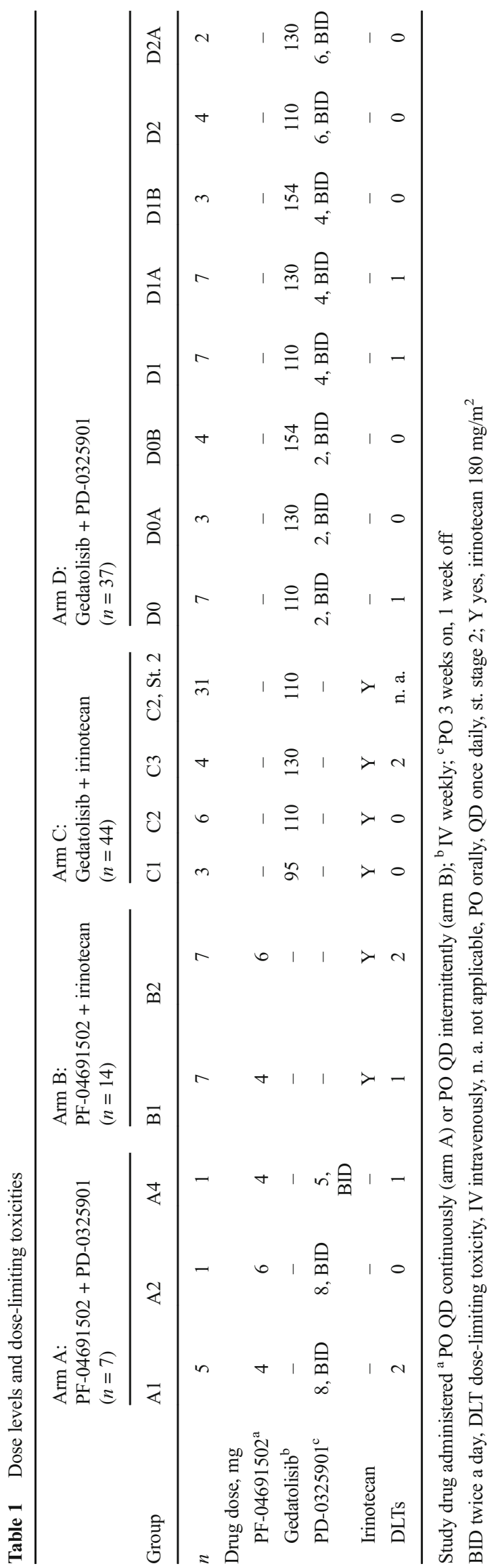

endpoints included evaluation of the safety profile of each combination, characterization of single- and multiple-dose PKs for gedatolisib, PD-0325901 and PD-0315209 (metabolite of PD-0325901) when administered in combination; assessment of the antitumor activity of the combinations; and evaluation of pretreatment tumor samples (fresh or archived) for genetic alterations that might predict tumor response.

The study was approved by the institutional review board or independent ethics committee of each participating center and followed the Declaration of Helsinki and International Conference on Harmonization Good Clinical Practice guidelines. All subjects gave written informed consent. The study was sponsored by Pfizer and registered at ClinicalTrials.gov (NCT01347866).

\subsection{Eligible Patients}

Patients 18 years of age or older with a histologic or cytologic diagnosis of advanced/metastatic solid tumor for which there was no current clinically effective treatment were included in the study. Patients had to have adequate bone marrow, renal, liver and cardiac functions, and adequate glucose control (including no previous diagnosis of diabetes mellitus and hemoglobin A1C $<7 \%$ ). Only patients with Eastern Cooperative Oncology Group performance status 0 or 1 were eligible. Patients enrolled in stage 2 (expansion cohort) of arm $\mathrm{C}$ were required to have metastatic CRC (regardless of KRAS mutation status), which had progressed on irinotecan-based regimens, or pancreatic ductal adenocarcinoma after progression on first-line treatment for advanced disease. Patients enrolled in stage 1 (dose escalation) of arm D were required to have tumors with KRAS or BRAF mutation (archived or fresh biopsy).

Patients with known active brain metastases, clinically decompensated cardiovascular disease, impaired pulmonary function, or surgery within 4 weeks prior to the start of study treatment were not eligible. Additionally, chemotherapy, radiotherapy and biologic or investigational anticancer therapy within 4 weeks prior to the start of study treatment were not allowed. Use of or anticipated need for cytochrome P450 3A4 inhibitors/inducers or drugs known to be UGT1A9 substrates, and concurrent administration of herbal preparations were also not permitted.

\subsection{Study Treatments}

Treatment schedules for arms A and B are summarized in Supplementary Information. In arm C, gedatolisib was dosed as an IV infusion weekly on days 2, 9, 16 and 23 of each 28day cycle; irinotecan $180 \mathrm{mg} / \mathrm{m}^{2}$ was administered IV on days 1 and 15 of each 28-day cycle. In arm D, gedatolisib was dosed as an IV infusion weekly on days -14 (during the PK 
lead-in portion of study), and on days 1, 8, 15 and 22 of each 28-day cycle. PD-0325901 was administered orally BID from day -7 to day -1 (during the PK lead-in portion of the study) and on days 1-21 of each 28-day cycle. Dose levels and patient groups for arms A to D are listed in Table 1.

In arm C, the starting dose was gedatolisib $95 \mathrm{mg}$, which was escalated using the classical $3+3$ design. Initially, up to three patients were to be treated at each dose level (DL). If a DLT was observed in one of the three initially treated patients, three additional patients were to be enrolled and treated at the same DL. A zone-based design was used for dose escalation in arm $\mathrm{D}$, consisting of a modified $3+3$ design that allowed opening more than one DL at the same time once DL0 had been completed. Dose escalation continued until an MTD (or two MTDs) was defined or the maximum allowable dose was reached. The starting dose levels for arm D (DL0) were gedatolisib $110 \mathrm{mg}$ and PD-0325901 $2 \mathrm{mg}$ BID (Table 1). Once it had been established that the DL0 (110 mg $+2 \mathrm{mg})$ was safe (no DLT in three patients or $\leq 1$ DLT in six patients), DL1 (110 mg $+4 \mathrm{mg})$ and DLA (130 mg $+2 \mathrm{mg})$ could be opened.

\subsection{Dose-Limiting Toxicity and Maximum Tolerated Dose}

A DLT was defined as any of the following AEs (classified according to the Common Terminology Criteria for Adverse Events [CTCAE] version 4.0) that were attributable to the combination study drug (i.e., deemed by the investigator to be at least possibly related) and occurring in the first cycle (28 days of treatment): (i) hematologic AEs: grade 4 neutropenia lasting $>7$ days, febrile neutropenia, grade $\geq 3$ neutropenic infection, grade 3 thrombocytopenia with bleeding, grade 4 thrombocytopenia; (ii) non-hematologic AEs: grade $\geq 3$ toxicities, except those that had not been optimally treated (i.e., nausea, vomiting, diarrhea, hyperglycemia); grade 3 corrected QT prolongation $>500$ msec persisting after correction of any reversible causes (i.e., electrolyte abnormalities or hypoxia); and persistent, intolerable toxicities (i.e., grade 2 mucosal inflammation, grade 2 neutropenia or grade 2 fatigue) resulting in failure to deliver at least $75 \%$ of doses during the first cycle or in a delay $>2$ weeks in starting cycle 2 . The MTD estimate was the highest dose associated with the occurrence of DLTs in $<33 \%$ of patients in the first treatment cycle, with the next higher dose having at least 2 of 3 to 6 patients experiencing DLTs.

\subsection{Safety and Antitumor Activity Assessments}

Safety evaluations included clinical monitoring, vital signs, physical examination, 12-lead electrocardiogram, cardiac function, collection of AEs and safety laboratory test results. The AEs were graded according to CTCAE v4.0 and coded using Medical Dictionary for Regulatory Activities v18.1.

Antitumor activity was evaluated through radiologic tumor assessments performed at baseline, every 8 weeks during treatment (cycle 3 and beyond), whenever disease progression was suspected (e.g., symptomatic deterioration) and at the time of withdrawal from the study (if not done in the previous 6 weeks) or at the end of treatment. Responses were determined using Response Evaluation Criteria in Solid Tumors (RECIST) version 1.1.

\subsection{Pharmacokinetics and Genetic Markers}

Blood samples were collected for PK analysis of PF04691502, gedatolisib, PD-0325901 and PD-0315209 (a metabolite of PD-0325901) at predefined time points and were assayed using validated analytical methods. The lower limit of quantification (LLOQ) was $0.25 \mathrm{ng} / \mathrm{mL}$ for PF04691502, $1 \mathrm{ng} / \mathrm{mL}$ for PD-0325901 and $2 \mathrm{ng} / \mathrm{mL}$ for gedatolisib. Samples below the LLOQ were set to zero for analysis.

Patient samples (formalin-fixed paraffin-embedded tumor tissues) were analyzed for genetic markers potentially correlated to drug sensitivity, including phosphatase and tensin homolog (PTEN) expression, KRAS/BRAF mutation status and PIK3CA mutation. PTEN protein expression was assessed by immunohistochemistry staining and reported as manual pathologist scores $(0,1+, 2+$ or $3+) . K R A S$ and $B R A F$ gene mutation analyses were performed using the Qiagen KRAS RGQ PCR assay (Qiagen, Manchester, UK). PIK3CA gene mutation analysis was performed using a Roche Cobas PIK3CA assay (Roche Molecular Diagnostics, Pleasanton, CA, USA).

\subsection{Statistical Analysis}

Descriptive statistics were used throughout the study. The objective response rate (ORR) was summarized with an exact two-sided 95\% confidence interval (CI) calculated using the Clopper-Pearson method. Progression-free survival (PFS), defined as the time from first dose of study treatment to first documentation of progression or death due to any cause, was analyzed by descriptive statistics and the Kaplan-Meier method. The PK parameters were calculated for each patient using non-compartmental analysis of concentration-time data.

\section{Results}

Seven and 14 patients were screened and received study treatment in arm A (three dose-escalation groups) and arm B (two dose-escalation groups), respectively (Table 1). A total of 45 patients were screened and 44 received study treatment in arm 
Table 2 Patient demographic and baseline characteristics

\begin{tabular}{|c|c|c|c|c|}
\hline & $\begin{array}{l}\text { Arm A: } \\
\text { PF- } \\
04691502+\text { PD- } \\
0325901\end{array}$ & $\begin{array}{l}\text { Arm B: } \\
\text { PF- } \\
04691502+\text { irinotecan }\end{array}$ & $\begin{array}{l}\text { Arm C: } \\
\text { Gedatolisib + } \\
\text { irinotecan }\end{array}$ & $\begin{array}{l}\text { Arm D: } \\
\text { Gedatolisib + } \\
\text { PD-0325901 }\end{array}$ \\
\hline$n$ & 7 & 14 & 44 & 37 \\
\hline \multicolumn{5}{|l|}{ Gender, $n(\%)$} \\
\hline Male & $4(57.1)$ & $7(50)$ & $25(56.8)$ & $22(59.5)$ \\
\hline Female & $3(42.9)$ & $7(50)$ & $19(43.2)$ & $15(40.5)$ \\
\hline \multicolumn{5}{|l|}{ Age, years } \\
\hline Mean (range) & $56.7(49-73)$ & $56.4(37-81)$ & $58.5(33-78)$ & $58.3(33-75)$ \\
\hline$\geq 65, n(\%)$ & $1(14.3)$ & $4(28.6)$ & $15(34.1)$ & $14(37.8)$ \\
\hline \multicolumn{5}{|l|}{ Race, $n(\%)$} \\
\hline White & $7(100)$ & $14(100)$ & $34(77.3)$ & $33(89.2)$ \\
\hline Black & 0 & 0 & $4(9.1)$ & 0 \\
\hline Asian & 0 & 0 & $5(11.4)$ & $4(10.8)$ \\
\hline Other & 0 & 0 & $1(2.3)$ & 0 \\
\hline \multicolumn{5}{|l|}{$\begin{array}{l}\text { Primary tumor } \\
\quad \text { diagnosis, } n(\%)\end{array}$} \\
\hline Colorectal cancer & $5(71.4)$ & $8(57.1)$ & $41(93.2)$ & $21(56.8)$ \\
\hline Lung cancer & 0 & 0 & $1(2.3)$ & $5(13.5)$ \\
\hline Ovarian cancer & 0 & $2(14.3)$ & 0 & $5(13.5)$ \\
\hline Pancreatic cancer & 0 & 0 & $2(4.5)$ & 0 \\
\hline Breast cancer & $1(14.3)$ & $1(7.1)$ & 0 & 0 \\
\hline $\begin{array}{r}\text { Gastrointestinal } \\
\text { stromal tumor }\end{array}$ & $1(14.3)$ & 0 & 0 & 0 \\
\hline Gastric cancer & 0 & $1(7.1)$ & 0 & $1(2.7)$ \\
\hline Cholangiocarcinoma & 0 & $1(7.1)$ & 0 & $1(2.7)$ \\
\hline $\begin{array}{l}\text { Esophageal } \\
\text { carcinoma }\end{array}$ & 0 & $1(7.1)$ & 0 & 0 \\
\hline Endometrial cancer & 0 & 0 & 0 & $1(2.7)$ \\
\hline Liposarcoma & 0 & 0 & 0 & $1(2.7)$ \\
\hline $\begin{array}{l}\text { Cancer of the } \\
\text { appendix }\end{array}$ & 0 & 0 & 0 & $1(2.7)$ \\
\hline Prostate cancer & 0 & 0 & 0 & $1(2.7)$ \\
\hline \multicolumn{5}{|c|}{$\begin{array}{l}\text { No. of prior systemic } \\
\quad \text { anticancer treatment(s), } n(\%)\end{array}$} \\
\hline 1 & 0 & $2(14.3)$ & $6(13.6)$ & $2(5.4)$ \\
\hline 2 & $3(42.9)$ & $4(28.6)$ & $8(18.2)$ & $10(27.0)$ \\
\hline 3 & $2(28.6)$ & $3(21.4)$ & $11(25.0)$ & $10(27.0)$ \\
\hline$>3$ & $2(28.6)$ & $5(35.7)$ & $19(43.2)$ & $15(40.5)$ \\
\hline
\end{tabular}

C: 13 patients in three dose-escalation groups/stage 1 and 31 patients in the expansion cohort/stage 2. In arm D, 39 patients were screened and 37 received study treatment (eight doseescalation groups/stage 1). Patient demographic characteristics and primary tumor diagnosis are summarized in Table 2. The majority of patients had a primary diagnosis of advanced CRC: $71 \%, 57 \%, 93 \%$ and $57 \%$ in arms A, B, C and D, respectively. All 31 patients in the expansion cohort of arm C (C2, stage 2) had advanced CRC. Five (14\%) patients each in the dose-escalation groups of arm D had advanced ovarian and lung cancer. Approximately $57 \%$ of the patients in arms A and $\mathrm{B}$, and $68 \%$ of patients in arms $\mathrm{C}$ and $\mathrm{D}$ had received three or more prior systemic anticancer treatment regimens. All patients have been discontinued from the study.

\subsection{Safety}

In arm A (PF-04691502 in combination with PD-0325901), DLTs were reported in three of the seven DLT-evaluable patients. In group A1, one patient experienced grade 3 diarrhea, grade 3 nausea and grade 3 vomiting not improved by symptomatic treatment; one patient developed grade 4 mucosal 
inflammation. In group A4, one patient experienced grade 3 increased blood alkaline phosphatase (Table 1 and Supplementary Table S1). In arm A, five (71.4\%) patients experienced grade 3-4 all-causality AEs and five (71.4\%) patients had grade 3-4 treatment-related AEs (Supplementary Tables S2 and S3).

In arm B (PF-04691502 in combination with irinotecan), DLTs were reported in three of the 12 DLT-evaluable patients. In group B1, one patient experienced grade 2 neutropenia; in $\mathrm{B} 2$, one patient reported grade 2 fatigue and one patient developed grade 3 febrile neutropenia (Table 1 and Supplementary Table S1; persistent AEs resulting in failure to deliver at least $75 \%$ of doses during the first cycle or in a delay $>2$ weeks in starting cycle 2 were considered DLTs). Among all patients in arm B $(n=14)$, seven $(50 \%)$ patients experienced grade 3-4 all-causality AEs and four (28.6\%) patients developed grade 3-4 treatment-related AEs (Supplementary Tables S2 and S4).

Both arms A and B of this study were closed prematurely because of safety concerns and greater antitumor activity seen in arms C and D. The MTDs for the combinations of PF04691502 with PD-0325901 or irinotecan were not estimated.

In arm C (gedatolisib in combination with irinotecan), all 13 patients in the dose-escalation stage were evaluable for DLT. DLTs were reported in two patients treated at the gedatolisib 130-mg dose level (group C3): one patient developed grade 4 febrile neutropenia and one patient experienced grade 3 fatigue (Table 1 and Supplementary Table S1). The MTD for gedatolisib in combination with irinotecan $180 \mathrm{mg} /$ $\mathrm{m}^{2}$ was estimated to be $110 \mathrm{mg}$ weekly.

Among all patients in arm C ( $n=44), 17(38.6 \%)$ patients experienced grade 3-4 all-causality AEs. Eleven (25.0\%) patients developed grade 3-4 treatment-related AEs, including grade 4 neutropenia $(n=3)$ and grade 4 febrile neutropenia $(n=1)$. Treatment-related grade 3 AEs experienced by two or more patients were neutropenia $(n=4)$ and asthenia $(n=2)$. The most common treatment-related AEs were nausea $(61.4 \%)$, diarrhea $(52.3 \%)$, vomiting $(40.9 \%)$, mucosal inflammation/stomatitis (34.1\%), decreased appetite (31.8\%) and fatigue (29.5\%), mostly of grade 1 or 2 (Table 3). Four (9.1\%) patients had all-causality AEs reported as the primary reason for permanently discontinuing treatment (Supplementary Table S2). Ten (22.7\%) patients temporarily discontinued gedatolisib due to all-causality AEs; three $(6.8 \%)$ of these discontinuations were due to treatmentrelated AEs. Eight (18.2\%) patients temporarily discontinued irinotecan due to all-causality AEs; two $(4.5 \%)$ of these discontinuations were due to treatment-related AEs. Irinotecan doses were reduced in $10(22.7 \%)$ patients due to treatmentrelated AEs.

In arm D (gedatolisib in combination with PD-0325901), DLTs were reported in three of the 35 DLT-evaluable patients.
One patient (group D0) experienced grade 2 mucosal inflammation, one patient (group D1) had grade 3 hypophosphatemia and one patient (group D1A) had grade 3 stomatitis (Table 1 and Supplementary Table S1). The MTD for combination treatment in arm D was not reached at the highest dose level of gedatolisib evaluated in this study (gedatolisib $154 \mathrm{mg}$ weekly/PD-0325901 $4 \mathrm{mg} \mathrm{BID).}$

In arm D, 19 (51.4\%) patients experienced grade 3-4 allcausality AEs; eight $(21.6 \%)$ patients had treatment-related grade 3-4 AEs, including grade 3 mucosal inflammation/ stomatitis $(n=3)$; increased alanine and aspartate aminotransferase levels, hypophosphatemia, macular rash and proteinuria ( $n=1$ each); and grade 4 hyperglycemia $(n=1)$ (Table 4$)$. The most common treatment-related AEs in arm D were mucosal inflammation/stomatitis (67.6\%), acneiform dermatitis $(40.5 \%)$, nausea $(35.1 \%)$, fatigue $(29.7 \%)$ and rash $(29.7 \%)$. Six $(16.2 \%)$ patients had all-causality AEs as the primary reason for permanently discontinuing treatment (Supplementary Table S2). Eighteen (48.6\%) patients temporarily discontinued gedatolisib due to all-causality AEs; 15 $(40.5 \%)$ of these discontinuations were due to treatmentrelated AEs. Seventeen (45.9\%) patients temporarily discontinued PD-0325901 due to all-causality AEs, of which $12(32.4 \%)$ were due to treatment-related AEs. Grade 5 AEs were reported only in arm D in three $(8.1 \%)$ patients (cardiac tamponade, hypercalcemia and abdominal pain in patients with disease progression) and deemed not related to study treatment (Supplementary Table S2).

\subsection{Antitumor Activity}

In arm $\mathrm{C}$, two patients with $\mathrm{CRC}$ ( $\mathrm{C} 1$ and $\mathrm{C} 2$ /stage 2 groups), treated with gedatolisib in combination with irinotecan, achieved a partial response (PR; i.e., at least a $30 \%$ reduction from nadir; Fig. 1a). Both responders had received irinotecan therapy for advanced disease prior to study entry. These two patients remained on treatment for 189 and 359 days from first dose, respectively. The ORR was 4.7\% (95\% CI: 0.6-15.8\%). Clinical benefit (defined as complete response [CR], PR or stable disease $\geq 184$ days) was seen in seven (16.3\% [95\% CI: $6.8-30.7 \%]$ ) patients. The median PFS, calculated only for patients in the expansion cohort/stage 2 of arm $\mathrm{C}$, was 2.8 (95\% CI: 1.7-3.7) months.

In arm D, three patients with ovarian cancer (D0A, D1 and D1A groups) and one patient with endometrial cancer (D1 group) treated with gedatolisib in combination with PD0325901 achieved a PR (Figure 1b), for an ORR of $11.1 \%$ (95\% CI: 3.1-26.1\%). These four patients remained on treatment for 153, 259, 244 and 376 days, respectively, from first dose. Clinical benefit was seen in five (13.9\% [95\% CI: $4.7-$ 29.5\%]) patients.

Among responders in both arms $\mathrm{C}$ and $\mathrm{D}$, five of the five PTEN-evaluable patients were $\mathrm{PTEN}^{+}$, four of the five $K R A S$ - 
Table 3 Treatment-related adverse events reported in $>15 \%$ of patients in arm C $(n=44)$

\begin{tabular}{lllllll}
\hline Adverse event, $n(\%)$ & Grade 1 & Grade 2 & Grade 3 & Grade 4 & Grade 5 & Total \\
\hline Any adverse event & $13(29.5)$ & $19(43.2)$ & $7(15.9)^{\mathrm{a}}$ & $4(9.1)^{\mathrm{b}}$ & 0 & $43(97.7)$ \\
Nausea & $19(43.2)$ & $8(18.2)$ & 0 & 0 & 0 & $27(61.4)$ \\
Diarrhea & $15(34.1)$ & $7(15.9)$ & $1(2.3)$ & 0 & 0 & $23(52.3)$ \\
Vomiting & $13(29.5)$ & $5(11.4)$ & 0 & 0 & 0 & $18(40.9)$ \\
Mucosal inflammation/stomatitis & $14(31.8)$ & $1(2.3)$ & 0 & 0 & 0 & $15(34.1)^{\mathrm{c}}$ \\
Decreased appetite & $10(22.7)$ & $4(9.1)$ & 0 & 0 & 0 & $14(31.8)$ \\
Fatigue & $7(15.9)$ & $5(11.4)$ & $1(2.3)$ & 0 & 0 & $13(29.5)$ \\
Alopecia & $8(18.2)$ & $2(4.5)$ & 0 & 0 & 0 & $10(22.7)$ \\
Neutropenia & 0 & $2(4.5)$ & $4(9.1)$ & $3(6.8)$ & 0 & $9(20.5)$ \\
Asthenia & $3(6.8)$ & $4(9.1)$ & $2(4.5)$ & 0 & 0 & $9(20.5)$ \\
Abdominal pain & $5(11.4)$ & $2(4.5)$ & 0 & 0 & 0 & $7(15.9)$ \\
\hline
\end{tabular}

${ }^{a}$ One patient each had grade 3 hyperglycemia, pyrexia, bacteremia (Escherichia), leucopenia and anemia

${ }^{\mathrm{b}}$ One patient had grade 4 febrile neutropenia

${ }^{\mathrm{c}}$ One patient had both mucosal inflammation and stomatitis which were reported as separate, treatment-related AEs. This patient is counted twice in the category of mucosal inflammation and stomatitis evaluable patients had a detectable $K R A S$ mutation in either ovarian (G12A, G12 V and G13D) or endometrial cancer (G12D) and one of the two phosphatidylinositol-4,5bisphosphate 3-kinase catalytic subunit alpha gene (PIK3CA)-evaluable patients had a detectable mutation (H1047R) in endometrial cancer. No $B R A F$ mutations were detected in the three $B R A F$-evaluable responders (Supplementary Table S5).

\subsection{Pharmacokinetics}

Gedatolisib PK profiles are presented for arm C (in combination with irinotecan) and arm D (in combination with PD0325901). Median plasma concentration-time profiles of gedatolisib in arms $\mathrm{C}$ and $\mathrm{D}$ are shown in Fig. 2. Plasma gedatolisib PK parameters during once-weekly administration are summarized in Supplementary Table S6 for arm C (cycle 1, days 2 and 16) and Supplementary Table S7 for arm D (lead-in and cycle 1, day 15). The range of gedatolisib doses administered was less than twofold, ranging from 95 to $130 \mathrm{mg}$ in arm C and from 110 to $154 \mathrm{mg}$ in arm D. Plasma gedatolisib PK parameters were generally similar across the different treatment groups in both arms $\mathrm{C}$ and $\mathrm{D}$, with a trend toward higher exposure (maximum plasma concentration and area under the plasma concentration-time profile) at higher doses, but with considerable overlap between the dose levels. Mean terminal half-life ranged from 24 to $41 \mathrm{~h}$.

The PK parameters for PD-0325901 in arm D are summarized in Supplementary Table S8 for lead-in (day -1) and cycle 1 , day 1 following oral doses of $2 \mathrm{mg}$ to $6 \mathrm{mg}$ BID starting on day -7 of the lead-in phase. The PK parameters were similar for both time points. Plasma PD-0325901
Table 4 Treatment-related adverse events reported in $>15 \%$ of patients in arm $\mathrm{D}(n=37)$

\begin{tabular}{lclllll}
\hline Adverse event, $n(\%)$ & Grade 1 & Grade 2 & Grade 3 & Grade 4 & Grade 5 & Total \\
\hline Any adverse event & $8(21.6)$ & $20(54.1)$ & $7(18.9)^{\mathrm{a}}$ & $1(2.7)^{\mathrm{b}}$ & 0 & $36(97.3)$ \\
Mucosal inflammation/stomatitis & $14(37.8)$ & $8(21.6)$ & $3(8.1)$ & 0 & 0 & $25(67.6)$ \\
Acneiform dermatitis & $11(29.7)$ & $4(10.8)$ & 0 & 0 & 0 & $15(40.5)$ \\
Nausea & $10(27.0)$ & $3(8.1)$ & 0 & 0 & 0 & $13(35.1)$ \\
Fatigue & $7(18.9)$ & $4(10.8)$ & 0 & 0 & 0 & $11(29.7)$ \\
Rash & $6(16.2)$ & $5(13.5)$ & 0 & 0 & 0 & $11(29.7)$ \\
Diarrhea & $7(18.9)$ & $3(8.1)$ & 0 & 0 & 0 & $10(27.0)$ \\
Maculopapular rash & $6(16.2)$ & $3(8.1)$ & 0 & 0 & 0 & $9(24.3)$ \\
Dry mouth & $8(21.6)$ & 0 & 0 & 0 & 0 & $8(21.6)$ \\
Vomiting & $5(13.5)$ & $3(8.1)$ & 0 & 0 & 0 & $8(21.6)$ \\
Increased aspartate aminotransferase & $3(8.1)$ & $2(5.4)$ & $1(2.7)$ & 0 & 0 & $6(16.2)$ \\
Asthenia & $2(5.4)$ & $4(10.8)$ & 0 & 0 & 0 & $6(16.2)$ \\
\hline
\end{tabular}

${ }^{\text {a }}$ One patient each had grade 3 increased alanine aminotransferase, hypophosphatemia, macular rash and proteinuria. ${ }^{\mathrm{b}}$ One patient developed grade 4 hyperglycemia 
Fig. 1 Waterfall plot of best response for patients in (a) arm C and (b) arm D
A

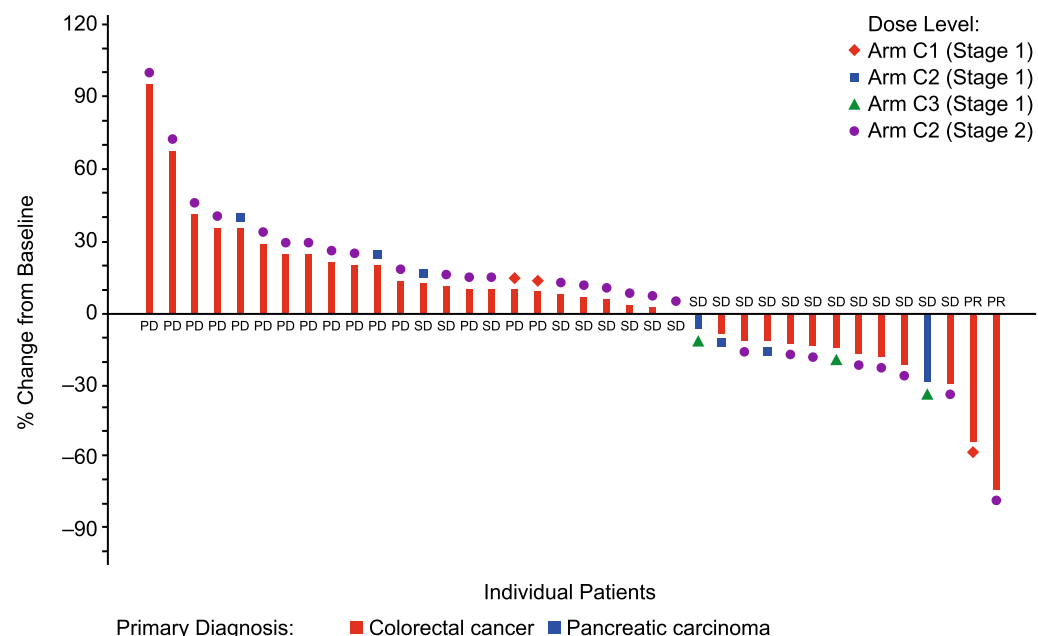

B

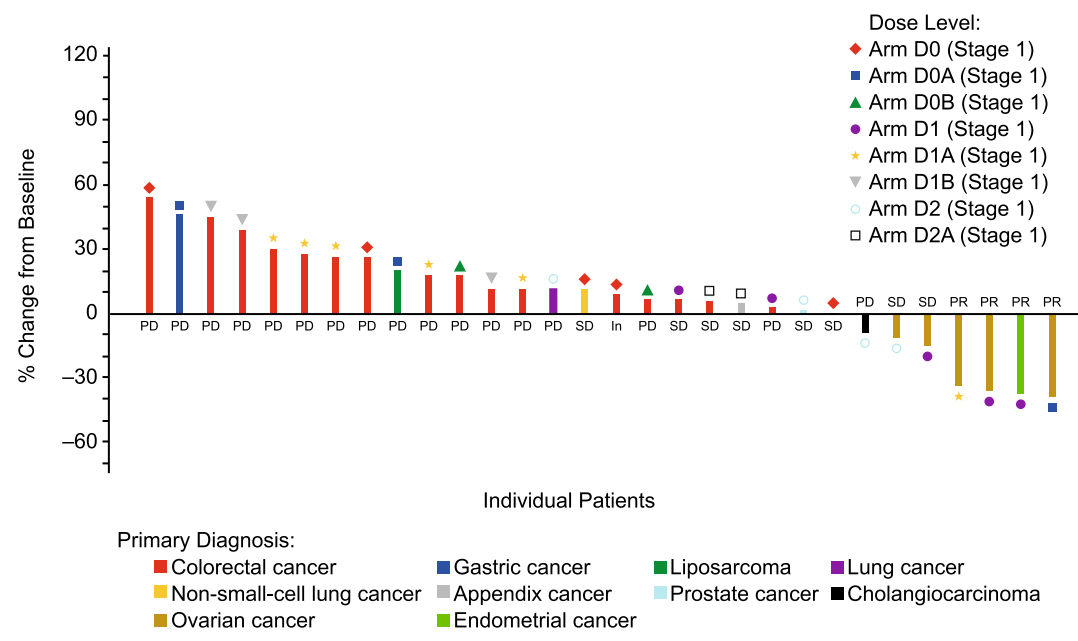

exposure (maximum concentration and area under the plasma concentration-time profile from time zero to $8 \mathrm{~h}$ post dose) appeared to increase proportionally with the twofold increase in dose from $2 \mathrm{mg}$ to $4 \mathrm{mg}$ BID; however, exposure for $6 \mathrm{mg}$ BID was similar to that for $4 \mathrm{mg}$ BID.

\section{Discussion}

In this multi-arm, multi-dose, phase I study we evaluated combinations of the PI3K/mTOR inhibitors PF-04691502 and gedatolisib with either irinotecan or the MEK inhibitor PD0325901 in the treatment of patients with advanced solid malignancies. The PF-04691502 arms A and B were closed prior to arms $\mathrm{C}$ and $\mathrm{D}$ due to the low tolerability profiles of the combinations investigated and the more encouraging antitumor activity observed in arms $\mathrm{C}$ and $\mathrm{D}$ with gedatolisib in combination with irinotecan or PD-0325901.
Arm C, unlike the other arms in this study, consisted of a fairly homogenous group of patients with advanced CRC. As one of the requirements of this arm was to enroll patients progressing on irinotecan-based therapies, the goal was to reverse the chemoresistance associated with irinotecan by adding a PI3K inhibitor. Similar strategies were successful in the early development of cetuximab in this indication [22]. In our study, the combination of gedatolisib with irinotecan induced a response rate of $\sim 5 \%$ and clinical benefit in $16 \%$ of patients, with a PFS of 2.8 months, which is consistent with previous reports in patients with refractory CRC [23, 24]. Furthermore, similar to other studies with PI3K inhibitors in metastatic CRC, there was no clear correlation between clinical benefit and the presence of activating PI3K mutations [25]. In our study, plasma concentrations of gedatolisib were generally similar across all dose groups in arm $\mathrm{C}$ (with irinotecan) and arm D (with PD-0325901). However, there was a trend toward higher exposure with higher doses across the dose range of $95 \mathrm{mg}$ to $154 \mathrm{mg}$ once weekly. This suggests 
Fig. 2 Median plasma concentration-time profile of gedatolisib in (a) arm C (cycle 1/day 16) and (b) arm D (cycle 1/day 15)

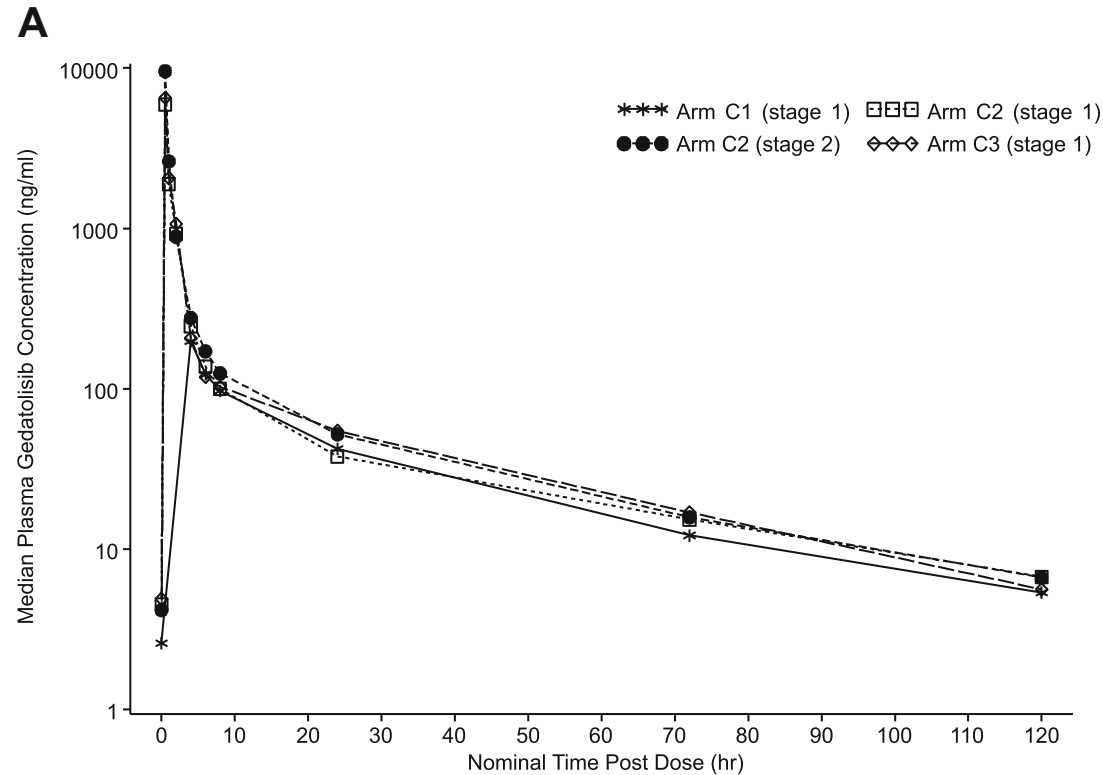

B

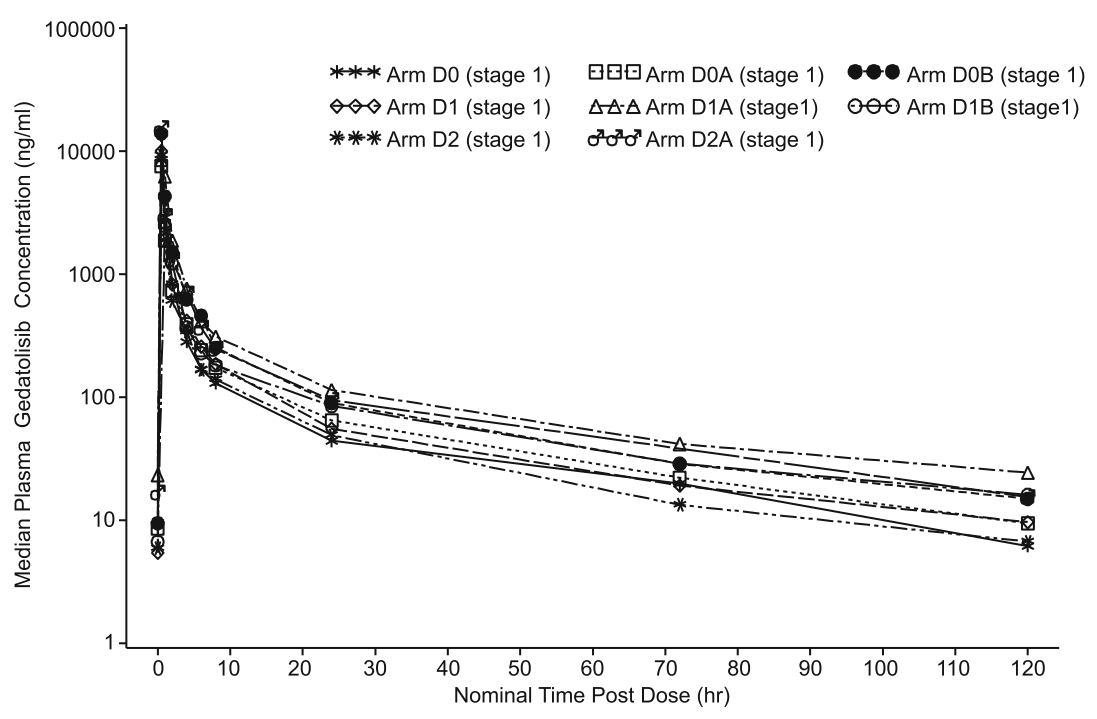

the combinations did reach active concentrations that affected target modulation in both study arms. However, frequent dose delays (for both gedatolisib and irinotecan) and dose reductions (for irinotecan) were required in arm $\mathrm{C}$ to manage the toxicities emerging during combination treatment. The most common AEs related to treatment with dual PI3K/mTOR inhibitors appeared to be primarily gastrointestinal in nature (e.g. diarrhea, nausea and vomiting), similar to the findings reported for dactolisib (BEZ235) in combination with everolimus in patients with advanced tumors [26].

In arm D, all patients enrolled had mutations in either $K R A S$ or $B R A F$ by trial design, suggesting potential clinical benefit in the combinations evaluated. Plasma PD-0325901 PK was similar across all treatment groups and plasma PD0325901 exposures appeared to increase proportionally with dose from $2 \mathrm{mg}$ to $4 \mathrm{mg}$ BID, whereas exposure for $6 \mathrm{mg}$ BID was similar to that of $4 \mathrm{mg}$ BID. In this arm, we observed responses and clinical benefit with gedatolisib in combination with PD-0325901 in patients with KRAS mutations, including patients with low-grade ovarian or endometrial cancer. Three of the five (60\%) patients with ovarian cancer achieved a PR and two (40\%) had stable disease, indicating clinical activity in this setting. The only patient with endometrial cancer enrolled in this study had a PR and a detectable PIK3CA H1047R mutation. This finding is consistent with prior phase II results, indicating clinical benefit from treatment with single-agent gedatolisib in $40 \%$ of patients $(n=38)$ with recurrent endometrial cancer (16\% ORR, one CR) [15]. Interestingly, other preclinical and clinical studies have previously suggested a potential association of the PIK3CA 
H1047R mutation with response to treatment with PI3K/AKT/mTOR inhibitors [25, 27].

Unfortunately, overall treatment in arm D was complicated by grade $>2$ AEs, which affected continuous dosing. Frequent dose delays were required for both combination drugs, which may have prevented therapeutic drug concentrations from being sustained over time. However, the combination of these two targeted therapies clearly has activity in KRAS-mutated ovarian cancer. Similarly, other trials evaluating the safety and efficacy of different combinations of PI3K and MAPK pathway inhibitors have shown antitumor activity, but the majority have been consistently associated with higher than expected toxicities $[12,28,29]$. Our trial was somewhat unique in that its main focus was in combination with chemotherapy, a different strategy from most other PI3K inhibitors in development. While no further development is currently in progress for PF-04691502 or gedatolisib in combination with irinotecan or PD-0325901, there are additional trials with gedatolisib in combination with cisplatin in triple-negative breast cancer to exploit the preclinical synergy seen when gedatolisib is combined with cytotoxic therapies.

Further investigations may help address the challenges encountered in the clinical development of PI3K inhibitors, with the identification of tolerable and effective schedules and combinations of PI3K inhibitors with other agents or chemotherapies. In addition, they may provide new insights into the apparent lack of consistent correlations of PI $3 \mathrm{~K} / \mathrm{mTOR}$ or pan-PI3K inhibitors effects with pathway aberrations $[25$, 30-33]. A new phase I study (NCT02684032) is currently in progress to evaluate gedatolisib in combination with the cyclin-dependent kinase 4/6 inhibitor palbociclib and either letrozole or fulvestrant in patients with estrogen receptor-positive/human epidermal growth factor receptor 2-negative advanced breast cancer.

Acknowledgments The authors thank the patients and their families/ caregivers, and the investigators, research nurses, study coordinators and operations staff who contributed to this study.

\section{Compliance with Ethical Standards}

Funding This study was sponsored by Pfizer Inc.

Conflict of Interest M. Alsina, I. Braña and G. Del Conte declare no potential conflicts of interest. Z. A. Wainberg received research funding from Novartis and Plexxikon; and honoraria from Array, Genentech and Sirtex. H. P. Soares received research funding and speaker honorarium from Novartis, consulting honoraria from Ipsen and has an advisory role for Cornerstone Pharmaceuticals. C. D. Britten received research funding and consulting honoraria from Pfizer. S. Leong and L. L. Siu received research funding from Pfizer. P. Ezeh, B. Houk, K. A. Kern, N. Pathan, K. J. Pierce and J. Vermette were employees of Pfizer during the conduct of this study. J. Tabernero had a consultant/advisory role for Amgen, Bayer, Boehringer Ingelheim, Celgene, Chugai, Genentech, Lilly, MSD, Merck Serono, Novartis, Roche, Sanofi, Symphogen, Taiho and Takeda. C. D.
Britten received support from the South Carolina Centers of Economic Excellence. Medical writing and editorial support was provided by $\mathrm{S}$. Mariani, MD, PhD, of Engage Scientific Solutions and funded by Pfizer Inc.

Open Access This article is distributed under the terms of the Creative Commons Attribution-NonCommercial 4.0 International License (http:// creativecommons.org/licenses/by-nc/4.0/), which permits any noncommercial use, distribution, and reproduction in any medium, provided you give appropriate credit to the original author(s) and the source, provide a link to the Creative Commons license, and indicate if changes were made.

\section{References}

1. Courtney KD, Corcoran RB, Engelman JA. The PI3K pathway as drug target in human cancer. J Clin Oncol. 2010;28(6):1075-83.

2. Roberts P, Der C. Targeting the Raf-MEK-ERK mitogen activated protein kinase cascade for the treatment of cancer. Oncogene. 2007;26(22):3291-310.

3. Arcila M, Lau C, Nafa K, Ladanyi M. Detection of KRAS and BRAF mutations in colorectal carcinoma. Roles for highsensitivity locked nucleic acid-PCR sequencing and broadspectrum mass spectrometry genotyping. J Mol Diagn. 2011;13(1):64-73.

4. Markman B, Atzori F, Pérez-García J, Tabernero J, Baselga J. Status of PI3K inhibition and biomarker development in cancer therapeutics. Ann Oncol. 2010;21(4):683-91.

5. Mendoza MM, Er EE, Blenis J. The RAS-Erk and PI3K-mTOR pathways: cross-talk and compensation. Trends Biochem Sci. 2011;36(6):320-8.

6. Braña I, Siu LL. Clinical development of phosphatidylinositol 3kinase inhibitors for cancer treatment. BMC Med. 2012;10:161-76.

7. Rodon J, Dienstmann R, Serra V, Tabernero J. Development of PI3K inhibitors: lessons learned from early clinical trials. Nat Rev Clin Oncol. 2013;10(3):143-53.

8. Simmons BH, Lee JH, Lalwani K, et al. Combination of a MEK inhibitor at sub-MTD with a PI3K/mTOR inhibitor significantly suppresses growth of lung adenocarcinoma tumors in Kras(G12D-LSL) mice. Cancer Chemother Pharmacol. 2012;70(2):213-20.

9. Britten CD. PI3K and MEK inhibitor combinations: examining the evidence in selected tumor types. Cancer Chemother Pharmacol. 2013;71(6):1395-409.

10. Pitts TM, Newton TP, Bradshaw-Pierce EL, et al. Dual pharmacological targeting of the MAP kinase and PI3K/mTOR pathway in preclinical models of colorectal cancer. PLoS One. 2014;9(11): e113037.

11. Mohan S, Vander Broek R, Shah S, et al. MEK inhibitor PD0325901 overcomes resistance to PI3K/mTOR inhibitor PF5212384 and potentiates antitumor effects in human head and neck squamous cell carcinoma. Clin Cancer Res. 2015;21(17):3946-56.

12. Jokinen E, Koivunen JP. MEK and PI3K inhibition in solid tumors: rationale and evidence to date. Ther Adv Med Oncol. 2015;7(3): $170-80$.

13. Yuan J, Mehta PP, Yin MJ, et al. PF-04691502, a potent and selective oral inhibitor of PI3K and mTOR kinases with antitumor activity. Mol Cancer Ther. 2011;10(11):2189-99.

14. Britten CD, Adjei AA, Millham R, et al. Phase I study of PF04691502, a small-molecule, oral, dual inhibitor of PI3K and mTOR, in patients with advanced cancer. Investig New Drugs. 2014;32(3):510-7. 
15. Del Campo JM, Birrer M, Davis C, et al. A randomized, phase II non-comparative study of PF-04691502 and gedatolisib (PF05212384) in patients with recurrent endometrial cancer. Gynecol Oncol. 2016;142(1):62-9.

16. Mallon R, Feldberg LR, Lucas J, et al. Antitumor efficacy of PKI587, a highly potent dual PI3K/mTOR kinase inhibitor. Clin Cancer Res. 2011;17(10):3193-203.

17. Wainberg ZA, Brega N, Davis C, et al. Randomized phase Ib/II study of PF-05212384 plus 5-fluorouracil-leucovorin-irinotecan (FOLFIRI) vs bevacizumab plus FOLFIRI in metastatic colorectal cancer (B2151007; NCT01937715). J Clin Oncol. 2014;32(15 suppl):TPS3657.

18. Tabernero J, Brega N, Davis C, et al. A randomized phase 2 study (B2151005) of PF-05212384 plus irinotecan versus cetuximab plus irinotecan in patients with wild-type KRAS metastatic colorectal cancer. J Clin Oncol. 2014;32(15 suppl):TPS3649.

19. Shapiro GI, Bell-McGuinn KM, Molina JR, et al. First-in-human study of PF-05212384 (PKI-587), a small-molecule, intravenous, dual inhibitor of PI3K and mTOR in patients with advanced cancer. Clin Cancer Res. 2015;21(8):1888-95.

20. LoRusso PM, Krishnamurthi SS, Rinehart JJ, et al. Phase I pharmacokinetic and pharmacodynamic study of the oral MAPK/ERK kinase inhibitor PD-0325901 in patients with advanced cancers. Clin Cancer Res. 2010;16(6):1924-37.

21. Haura EB, Ricart AD, Larson TG, et al. A phase II study of PD0325901, an oral MEK inhibitor, in previously treated patients with advanced non-small cell lung cancer. Clin Cancer Res. 2010;16(8): 2450-7.

22. Saltz LB, Lenz HJ, Kindler HL, et al. Randomized phase II trial of cetuximab, bevacizumab, and irinotecan compared with cetuximab and bevacizumab alone in irinotecan-refractory colorectal cancer: the BOND-2 study. J Clin Oncol. 2007;25(29):4557-61.

23. Lenz H-J, Van Cutsem E, Khambata-Ford S, et al. Multicenter phase II and translational study of cetuximab in metastatic colorectal carcinoma refractory to irinotecan, oxaliplatin, and fluoropyrimidines. J Clin Oncol. 2006;24(30):4914-21.
24. Lim R, Sun Y, Im SA, et al. Cetuximab plus irinotecan in pretreated metastatic colorectal cancer patients: the ELSIE study. World J Gastroenterol. 2011;17(14):1879-88.

25. Janku F, Hong DS, Fu S, et al. Assessing PIK3CA and PTEN in early-phase trials with PI3K/AKT/mTOR inhibitors. Cell Rep. 2014;6(2):377-87.

26. Wise-Draper TM, Moorthy G, Salkeni MA, et al. A phase Ib study of the dual PI3K/mTOR inhibitor dactolisib (BEZ235) combined with everolimus in patients with advanced solid malignancies. Target Oncol. 2017;12(3):323-32.

27. Janku F, Wheler JJ, Naing A, et al. PIK3CA mutation H1047R is associated with response to $\mathrm{PI} 3 \mathrm{~K} / \mathrm{AKT} / \mathrm{mTOR}$ signaling pathway inhibitors in early-phase clinical trials. Cancer Res. 2013;73(1): 276-84.

28. Bedard PL, Tabernero J, Janku F, et al. A phase Ib dose-escalation study of the oral pan-PI3K inhibitor buparlisib (BKM120) in combination with the oral MEK1/2 inhibitor trametinib (GSK1120212) in patients with selected advanced solid tumors. Clin Cancer Res. 2015;21(4):730-8.

29. Grilley-Olson JE, Bedard PL, Fasolo A, et al. A phase Ib doseescalation study of the MEK inhibitor trametinib in combination with the PI3K/mTOR inhibitor GSK2126458 in patients with advanced solid tumors. Investig New Drugs. 2016;34(6):740-9.

30. Dienstmann R, Serpico D, Rodon J, et al. Molecular profiling of patients with colorectal cancer and matched targeted therapy in phase I clinical trials. Mol Cancer Ther. 2012;11(9):2062-71.

31. Papadopoulos KP, Tabernero J, Markman B, et al. Phase I safety, pharmacokinetic, and pharmacodynamic study of SAR245409 (XL765), a novel, orally administered PI3K/mTOR inhibitor in patients with advanced solid tumors. Clin Cancer Res. 2014;20(9):2445-56.

32. Rodon J, Braña I, Siu LL, et al. Phase I dose-escalation and expansion study of buparlisib (BKM120), an oral pan-class I PI3K inhibitor, in patients with advanced solid tumors. Investig New Drugs. 2014;32(4):670-81.

33. LoRusso PM. Inhibition of the PI3K/AKT/mTOR pathway in solid tumors. J Clin Oncol. 2016;34(31):3803-15. 